\title{
ELECTROPHORESIS PRODUCTS
}

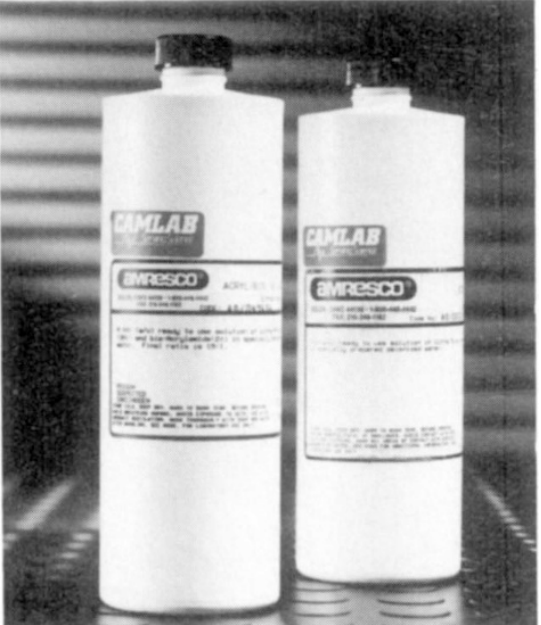

\section{Just Add Water}

The liquid acrylamide products from Camlab (Cambridge, U.K.) are premixed, ready-to-use solutions. Forty percent acrylamide, two percent bis-acrylamide and 19:1, 29:1, and 37.5:1 acrylamide:bis-acrylamide mixtures are available. Preparation requires only the addition of deionized water or buffers.

Write in 801 on Reader Service Card

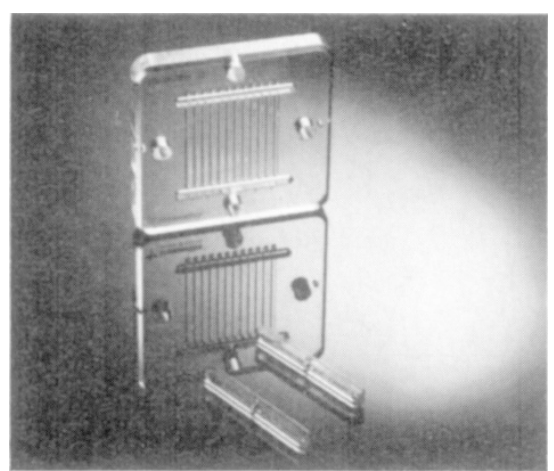

Stop Cutting Western Blots

New from Integrated Separation Systems (Hyde Park, MA), the Miniblotter 12 for screening antibodies and/or antigens with minimal amounts of reagents is specially engineered to match the lanes in intermediate Daiichi Gels and Mini-Sepragels. Antigen can be run in the 12 lanes of the intermediate gel, and those lanes can then be matched exactly to the channels of the Miniblotter 12. There is no need to cut the membrane. The Miniblotter 12 saves money by requiring minimal amounts of screening reagents, and saves time with its manifold which enables washing of all channels simultaneously.

Write in 806 on Reader Service Card

\section{Non-Acrylamide Gels}

J.T. Baker (Phillipsburg, NJ) now offers HydroLink D600, a non-acryl- amide monomer combined with an innovative cross-linker, for separating oligonucleotides and low-molecular-weight dsDNA. Hydrolink D600 directly substitutes for acrylamide-bis stock solutions. With high resolution, D600 yields up to one base resolution of nucleotides down to ten mers, including low MW PCR products, and up to four base-pair resolution of dsDNA fragments for oligonucleotide separation in the $10-300 \mathrm{bp}$ range. It can also be used in mini-gel format with shorter run times for oligonucleotide separation. DNA from D600 gels can be electroblotted and denatured in one hour. DNA can be electroeluted from D600 in one hour or salt eluted in 2-4 hours.

Write in 808 on Reader Service Card

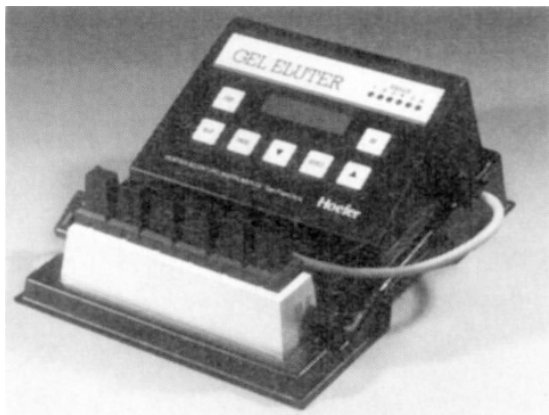

Freedom from Membranes

Hoefer Scientific's (San Francisco, CA) new GE 200 Mighty Fast Gel Eluter allows for 80-100 percent recovery of purified protein samples with fewer steps. The GE 200 utilizes two microcentrifuge tubes as buffer reservoirs; no dialysis membranes or high salt buffers are required. A 0.5 $\mathrm{ml}$ tube containing the sample to be eluted nests inside a standard $1.5 \mathrm{ml}$ tube. The electroeluted sample is collected in as little as $100-300 \mu \mathrm{l}$ of buffer. With sample cooling channel, a built-in $100 \mathrm{~V}$ power supply, and a user programmable microprocessor, the GE 200 can elute up to six samples at one time.

Write in 805 on Reader Service Card

\section{Gel Analysis}

The extended version of SW2000 from Ultra-Violet Products (Cambridge, U.K.) is a software image system for the evaluation of stained gels, autoradiographs, and TLC plates. Analysis can now be performed with or without base pair/molecular weight standards with the area of each peak represented either as an amount, a percentage, or a total amount. The new graphics section of the program allows the display of graphic overlays, gel images, or combinations of the two. A utilities menu also enables archiving and ready file access.

Write in 802 on Reader Service Card

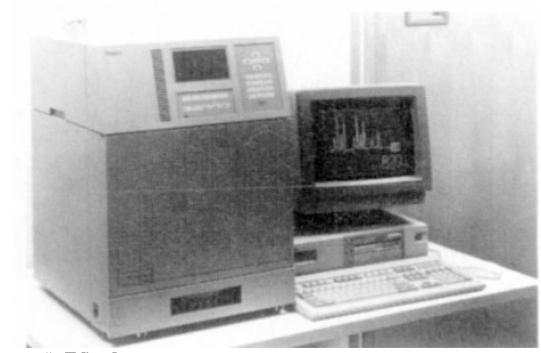

LC Unit

The Waters Division of Millipore (Watford, U.K.) has introduced the LC Module 1, an HPLC system. Its pumping unit can be used to blend up to four solvents, enabling the formation of isocratic mobile phases or gradients. Wavelengths can be switched during a run to provide a flexible approach, and up to 96 samples can be loaded automatically for rapid routine analyses. Operating parameters can be programmed via a cursor-guided menu and up to 15 different methods can be stored.

Write in 803 on Reader Service Card

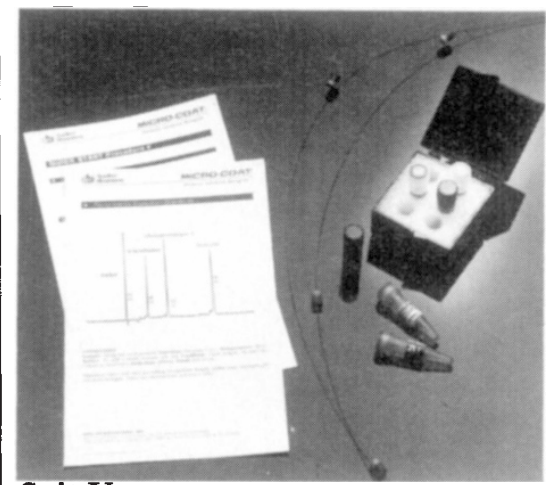

\section{Suit Up}

Applied Biosystems (Foster City, CA) announces the Micro-Coat Protein Analysis Kit for Applied Biosytems capillary electrophoresis. MicroCoat provides a fast simple procedure for analyzing a broad range of proteins in a wide $\mathrm{pH}$ range. Coating the capillary wall with Micro-Coat Protein Analysis Reagent produces a positively charged surface, permitting free migration through the capillary and subsequent detection. Micro-Coat offers minimal prerun preparation, direct detection, and nondestructive sample analysis. Kit includes the Protein Analysis Reagent, capillaries, a neutral marker, standards, and a protocol.

Write in 804 on Reader Service Card 